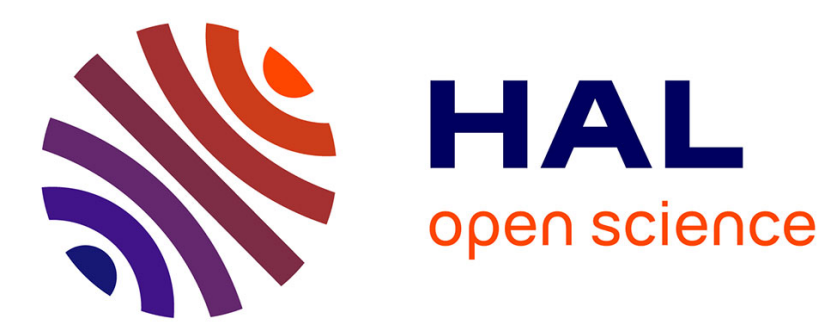

\title{
The Case for P2P Mobile Video System over Wireless Broadband Networks: A Practical Study of Challenges for a Mobile Video Provider
}

Yi Sun, Yang Guo, Xiaobing Zhang, Zhenyu Li, Kavé Salamatian

\section{- To cite this version:}

Yi Sun, Yang Guo, Xiaobing Zhang, Zhenyu Li, Kavé Salamatian. The Case for P2P Mobile Video System over Wireless Broadband Networks: A Practical Study of Challenges for a Mobile Video Provider. IEEE Network, 2013, 27 (2), pp.22-27. 10.1109/MNET.2013.6485092 . hal-00813588

\section{HAL Id: hal-00813588 \\ https://hal.science/hal-00813588}

Submitted on 15 Apr 2013

HAL is a multi-disciplinary open access archive for the deposit and dissemination of scientific research documents, whether they are published or not. The documents may come from teaching and research institutions in France or abroad, or from public or private research centers.
L'archive ouverte pluridisciplinaire HAL, est destinée au dépôt et à la diffusion de documents scientifiques de niveau recherche, publiés ou non, émanant des établissements d'enseignement et de recherche français ou étrangers, des laboratoires publics ou privés. 


\title{
The case for P2P Mobile Video System over wireless networks: A practical study of Challenges for a mobile video provider
}

\author{
Yi Sun, Yang Guo, Xiaobing Zhang, Zhenyu Li and Kave Salamatian
}

\begin{abstract}
Mobile video is becoming extremely popular these days and P2P mobile video platforms are considered for large deployment in this context. However, mobile networks have some peculiar characteristics that have to be considered for the design and deployment of realistic P2P mobile video system over a real mobile network. In this article, we take the viewpoint of a large-scale commercial P2P mobile video provider system, PPTV, and describe the challenges to implement a P2P mobile video system over 3G. Our analysis is backed by real measurement and experience from PPTV. We extract from these measurements the characteristics of mobile videos and analyze their impacts on P2P video systems. We also discuss other encountered practical problems in the design of a mobile P2P system for PPTV.
\end{abstract}

Index Terms-P2P, mobile video, PPTV.

\section{INTRODUCTION}

W ITH the steady increase in the number of mobile devices in usage worldwide, we witness a fast growing demand for mobile videos. According to Cisco Visual Networking Index [1], mobile video traffic exceeded 50 percent of the entire mobile data traffic in 2011 (597 petabytes per month) and will observe a 25 -fold increase at 2016 horizon. The popularity of mobile videos results from the combination of three factors: the development of wireless communication technology; the large availability of versatile and highly flexible mobile terminals platforms, such as smartphones and tablets, that are becoming more and more video-friendly; and finally the emergence of major video content providers, like YouTube, Netflix, Hulu, and PPTV that provide access to a huge catalogue of attractive video contents. The conjunction of the three above factors has enabled a ubiquitous access of users that can enjoy on their mobile devices, phone or tablet, their favorite videos wherever they are and whenever they wish.

While Video on Demand diffusion (VoD) over Internet has become an extremely popular service over a short time, a relatively large body of research has been devoted to understand its characteristics [2-5]. However, these works mainly studied non-mobile scenarios, while mobile devices have particular constraints and limitations (processor capacity, bandwidth, memory, energy capacity, etc.) that make it impossible to simply extend conclusions drawn on non-mobile scenarios to mobile environment, and to implement blindly solutions

Yi Sun, Yang Guo and Zhenyu Li are with Institute of Computing Technology, Chinese Academy of Sciences, Beijing, P. R. China. (e-mail: \{sunyi, guoyang, zyli\}@ict.ac.cn).

Xiaobing Zhang is with Shanghai Synacast Media Tech. CO. LTD (PPLive), Shanghai, P. R. China.

Kave Salamatian is with University of Savoie France, Paris, France. developed for wired Internet to mobile Internet. In particular, while peer-to-peer (P2P) TV has found its way into the video diffusion market over Internet by reducing the load on servers and better adapting to network capacity changes, mobile video services are still based on client-server approach. The major difference between a P2P system and a client-server approach is indeed that a P2P system is being composed of a number of nodes, each of which functions simultaneously as client and server, i.e., each node in a P2P network both downloads data from some nodes and uploads data to some other nodes. This prominent feature of $\mathrm{P} 2 \mathrm{P}$ networks helps the video server to reduce its upstream traffic and improve the video delivery performance, resulting in lower cost for the content provider and better performance for the final user. Introducing P2P technology into mobile video systems entails leveraging on the peers' uploads. As we will see later, the mandatory uploads make the introduction of $\mathrm{P} 2 \mathrm{P}$ approach in $3 \mathrm{G}$ mobile networks more challenging, if not impossible.

The aim of this article is to explore the challenges of using a $\mathrm{P} 2 \mathrm{P}$ paradigm for mobile video distribution over commercial wireless broadband network, like $3 \mathrm{G}$ or $4 \mathrm{G}$, from the viewpoint of a large-scale commercial $\mathrm{P} 2 \mathrm{P}$ mobile video provider system, PPTV. PPTV [6], also referred as PPLive, is currently the largest commercial online video service in China, and the largest media platform worldwide, offering both online live and On-Demand video broadcasting. This analysis will be backed by real measurements and feedback from PPTV design team that has the experience of running PPTV mobile video distribution platform. It is noteworthy that our main target in this paper is the P2P-based mobile video distribution over wireless broadband wireless, that puts aside WiFi based wireless networks where the problems are different.

We will first present some observations about the characteristics of mobile videos viewed through $3 \mathrm{G}$ coming from a comprehensive measurement of PPTV mobile diffusion platform and describe the impacts on $\mathrm{P} 2 \mathrm{P}$ transfer mode of these observations. We will thereafter focus on practical problems encountered during the development of $\mathrm{P} 2 \mathrm{P}$ mobile solutions and deployment process of it in PPTV. The rest of the article is organized as follows. In section II, we will give an overview to the PPTV dataset. Section III analyzes the dataset and present main observation made on it. We will further discuss the challenges we faced when designing a real large-scale P2P mobile video system in section IV. Section V presents our conclusion. 
TABLE I

DATASET STATISTICS

\begin{tabular}{|c|c|c|c|c|c|}
\hline \multirow{2}{*}{$\begin{array}{c}\text { Access } \\
\text { Method }\end{array}$} & \multicolumn{5}{|c|}{ Percentage of views from } \\
\cline { 2 - 6 } & $\mathrm{iPad}$ & iPhone & $\mathrm{aPad}$ & aPhone & others \\
\hline $3 \mathrm{G}$ & 1.02 & 73.84 & 4.35 & 20.78 & $\sim 0$ \\
\hline $\mathrm{WiFi}$ & 40.86 & 43.52 & 4.03 & 11.58 & 0.01 \\
\hline $\mathrm{WiFi+3G}$ & 37.91 & 45.57 & 4.17 & 12.34 & 0.01 \\
\hline
\end{tabular}

\section{DATASET DESCRIPTION}

The dataset we have used came from a comprehensive measurement of PPTV. As said before, PPTV offers both online live and On-Demand video streaming. The platform currently provides access to approximately 100 million video clips and 10,000 video content search records, with a library of more than 200,000 licensed movies and TV shows. Currently, PPTV has three different diffusion platforms: an HTTP-based website, a PC client software and some mobile applications for different platforms. The content distribution relies on a provisioned hybrid CDN-P2P content distribution network.

Our dataset is extracted from the logs of the PPTV mobile platform that covers over $120 \mathrm{TV}$ broadcasting stations, 300 live and 20,000 VoD channels. In between Dec. 1st, 2011 and Dec. 14th, 2011, we sampled uniformly the logs and gathered the logs of a total of 111,702,242 views, out of which $108,851,161$ views were VoD requests. By filtering out the logs with unknown access method, we obtained 86,521,403 video viewing logs, corresponding to $3,759,129$ users watching 427,316 unique videos. Among these view $92.58 \%$ were accessed via $\mathrm{WiFi}$ and $7.42 \%$ via $3 \mathrm{G}$.

An overview of the dataset is shown in Table I. We stratified the viewing sessions into different categories according to their access methods ( $3 \mathrm{G}$ or $\mathrm{WiFi}$ ) and terminal types (smartphones or pads). It is noteworthy that almost all viewing records come from IOS (iPad and iPhone) and Android (aPad and aPhone) devices. While for WiFi accesses, the number of views from smartphones and tablets is similar; for $3 \mathrm{G}$ accesses, most of the views are from smartphones. Overall, iPad and iPhone devices have generated much more views than Android pad and smartphones. These observations are somewhat different from other observations in U.S. and Western Europe, where the market share of Android in video and data consumption was observed to be equivalent to iPhone consumption [1]. This might be coming from the particular device market in China. As explained before the main target of our study in this paper is $3 \mathrm{G}$ networks.

\section{LESSONS LEARNED FROM THE DATASET}

In this section, we describe two major performance indicators obtained over measurements of PPTV mobile platform and we investigate their impacts on P2P transfer mode.

\section{A. Viewing Time}

We show in Fig. 1 the empirical distribution of the viewing time (normalized number of views vs. viewing time) for the two different access methods. Surprisingly a considerable large
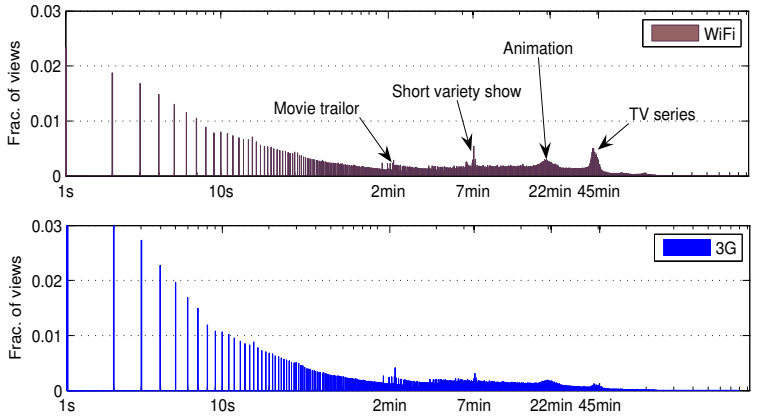

Fig. 1. Viewing time for different access method.

TABLE II

IMPACTS OF DEVICES ON VIEWING TIME (WIFI)

\begin{tabular}{|c|c|c|}
\hline Device & 50th percentile (s) & 95th percentile (min) \\
\hline iPad & 121 & 45.1 \\
\hline iPhone & 44 & 34.7 \\
\hline aPad & 80 & 45.8 \\
\hline aPhone & 58 & 42.8 \\
\hline
\end{tabular}

fraction of views last less than 10 seconds, and the number of views decreases with the longer viewing time. The reason is that mobile users are cautious about their data traffic and energy consumption and leave fast if the video is not what they expected. However for WiFi, we can observe some peaks in the long tail of viewing time distribution, corresponding to typical length for some video types, like movie trailer and TV series. These peaks are not observed for $3 \mathrm{G}$ users, meaning a fundamental difference between $3 \mathrm{G}$ and $\mathrm{WiFi}$ mobile users as WiFi users view this kind of videos, while $3 \mathrm{G}$ users rarely finish watching a full video. Additionally, we compared the distribution of viewing time over different devices. We show in Table II the median (50-percentile) and the 95-percentile of these devices. These results confirm that users using pads view longer videos online than those using phones. This can be easily explained by the fact that pads have larger screens and higher processing capacity, resulting in better watching experience.

\section{B. User Leaving Rate}

We give in Fig. 2 the distributions of the completion rate for video contents that has been viewed by the users stratified in three different class of video lengths: $0 \sim 10 \mathrm{~min}, 20 \sim 30 \mathrm{~min}$ and 40 50 min and two class of access to PPTV: using stand alone client and mobile application. On the $y$-axis, we show the relative number of users on the right, and the Cumulative Distribution Function (CDF) on the left whereas $x$-axis depicts the video completion rate. We can see that video length has a clear impact on the video completion rates, especially for mobile users. Users tend to watch more than half of the short videos while the completion rates of long videos rarely exceed 0.7. Compared with client users, mobile users generally have lower completion rates, with a high percentage of them only watching less than $25 \%$ of the videos. In particular when 

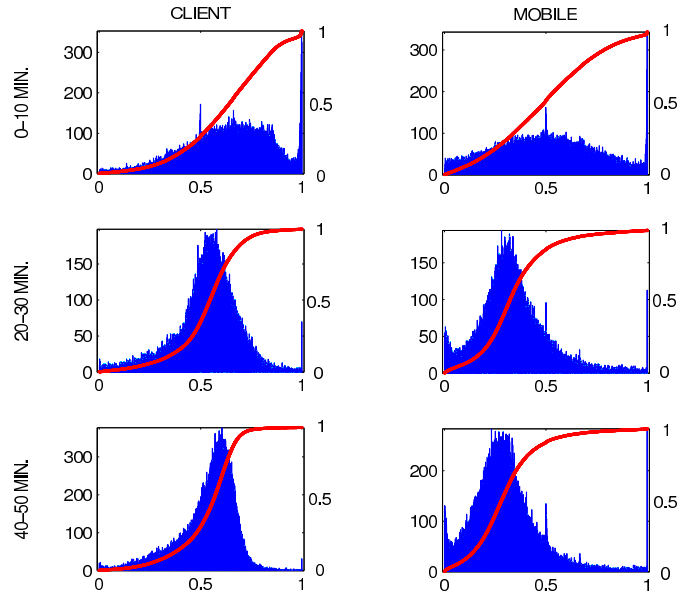

Fig. 2. Distributions of video completion rate.

video length exceeds 20 minutes, mobile users' distributions are shifted left below a completion rate of 0.5 which means that lots of mobile users leave at the first half of the videos.

\section{Analysis}

The above observations show that designing a mobile $\mathrm{P} 2 \mathrm{P}$ video system would be more challenging than a traditional one. First, compared with client-server mode, P2P requires more time to establish connection between nodes. However, as user viewing time is very short in mobile platform, this connection time becomes comparable with the patience limit of the user before leaving. Moreover in mobile environment, users usually do not finish watching a whole video. This results in availability of a very limited number of copies of the latter parts of the video file in the network. P2P downloading performance heavily depends on the number of resource copies in the network, and less video copies hinder greatly the usability of P2P system.

The two above observation advocates for the usage of a CDN based approach for mobile video distribution. CDN networks will guarantee a low connection delay in the beginning and the availability of latter parts of the video for the mobile users. Indeed one can consider using an internal P2P distribution system for the CDN in order to provide a better user experience. However, this P2P network will not be able to leverage on the mobile users.

\section{Practical Issues for Designing a Real LARGE-SCALE P2P Mobile Video SySTEM}

In the previous sections, we used insights coming from real measurements of PPTV to uncover some major challenges for the usability of P2P approaches for mobile video diffusion. This section will discuss some practical issues for building such large-scale $\mathrm{P} 2 \mathrm{P}$ mobile video system that results from our experience in the operating and designing PPTV.

\section{A. Connection Establishment}

Due to the massive amount of mobile terminals in a $3 \mathrm{G}$ network, there are not enough unique IPv4 addresses for each mobile terminal. So a local, private and non-routable IP address is generally assigned to a mobile device connected to the $3 \mathrm{G}$ network. These private IP addresses are to be translated to public IP addresses by Network Address Translation (NAT) gateway. However, NAT devices break end-to-end connectivity, as users outside the local network cannot directly access nodes behind a NAT. Establishing and maintaining IP connectivity with terminals behind NAT devices is called NAT traversal [7]. Indeed a major solution to this issue is a full migration of $3 \mathrm{G}$ network operator to IPv6. However this migration has still a long way to go, meaning that a mobile video distribution platform has still to consider NAT traversal.

The local NAT of a node A connected to a $3 \mathrm{G}$ network has to translate A's local IP address to a public IP address and maintain the status of this transformation, for enabling it to connect with a node $\mathrm{B}$. If the destination node $\mathrm{B}$ is also behind a NAT, a NAT traversal has to be implemented. After establishing the connection, the NAT devices use their local transformation tables to find the corresponding device A and $\mathrm{B}$ and deliver the data directed to them.

Two typical scenarios happen for NAT traversal in mobile P2P environment: (a) a host with a public address in the Internet wish to connect to a mobile devices; (b) two mobile devices connect to each other.

In $3 \mathrm{G}$ networks and more generally all wireless broadband networks, NAT boxes are deployed by mobile ISPs and generally centralized to simplify mobile node management. These boxes have to maintain a large number of sessions and IP address transformation status coming from the thousands of hundreds of devices in the $3 \mathrm{G}$ network. NAT maintenance is by itself a major challenge for mobile ISPs. The situation is likely to worsen if $\mathrm{P} 2 \mathrm{P}$ technology is used in a mobile network, as each mobile terminal will have to maintain several connections with different nodes to download and upload data and by result the number of sessions in the NAT devices will become even larger. According to PPTV experience, sometimes the mobile network ISPs block connections to reduce the burden on the NAT servers. This brings new obstacle for mobile P2P applications over wireless broadband networks.

The above-described issue is less acute in $\mathrm{WiFi}$ and other traditional networks as NAT boxes are completely distributed and each one covers a limited area with limited number of devices.

\section{B. 3G Network Business Model}

The business model of $3 \mathrm{G}$ traffic is generally different from classical IP connectivity. While generally IP connectivity is paid through a flat rate for an unlimited upload and/or download, customers pay $3 \mathrm{G}$ data traffic per upload/download consumption or have a limited allowance for upload/download. WiFi users also follow generally a flat rate (or even free when they connect to a free hotspot) and unlimited model. This business model generates major issues for P2P networks. It is required by users to do both upload and download in a P2P network, however as people have to pay for the uploading traffic that has not a direct benefit for them, they are likely to stop the uploading and become leechers, reducing significantly 
TABLE III

USER INTEREST ON VIDEOS

\begin{tabular}{|c|c|c|c|}
\hline & Web $(\%)$ & Client $(\%)$ & Mobile $(\%)$ \\
\hline Movie & 17 & 18 & 23 \\
\hline TV Show & 64 & 52 & 41 \\
\hline Cartoons & 11 & 19 & 22 \\
\hline Arts \& Entertainment & 4 & 6 & 11 \\
\hline Special Focus & $<1$ & 1 & $<1$ \\
\hline Game & 1 & 3 & 1 \\
\hline Sports & 2 & 1 & $<1$ \\
\hline
\end{tabular}

the number of resources copies in P2P network, and resulting in the P2P network to be inefficient [8]. One possible way to get around this issue would be to let mobile users only download contents and make fixed users of a P2P network to upload content. However, this trick will not be very effective. According to our analysis of PPTV logs, we show in Table III, video interests of users connected through different platforms. It can be seen that users of different platforms have different interests. Therefore, videos favored by $3 \mathrm{G}$ users may not have enough copies in the fixed-line network, resulting in a poor downloading performance for $3 \mathrm{G}$ users.

One potential solution will be for the P2P video content provider to implement a push approach that consisting in pushing some content to wired users in the network even if they did not request them in order to provide them for mobile (or other) users. By doing so the provider ensures there is enough copies available in the network. However, this approach is unfair to wired users that have to give a service to mobile users without any benefit in return and will therefore want to stop the pushed traffic by the content provider.

In conclusion, because of the current business model of $3 \mathrm{G}$ networks, mobile users might not be willing to upload data, as it will cost them directly. This appears to be a major barrier to the widespread of P2P mobile video systems.

\section{Device Capacities and Abilities}

Even if mobile devices are becoming more similar to traditional PCs, however, there are still some fundamental differences between mobile and traditional computer devices. Mobile devices are still limited in terms of storage, bandwidth, computing capacity, battery, etc. In the following, we will take a look at the impact of these limits on mobile P2P networks.

One of the first issues for mobile devices is memory storage. Even if typical storage of smartphones and pads are nowadays respectively, $4 \mathrm{G} \sim 16 \mathrm{G}$ and $16 \mathrm{G} \sim 64 \mathrm{G}$, the volume of information in form of image, music, applications that are stored on them is also steadily growing. P2P applications usually need $1 \mathrm{G} \sim 2 \mathrm{G}$ of free spaces for content buffer that is still considerable for mobile terminals. Nonetheless, a more important limitation exists; the current flash storage technology used for storage in mobile terminal is limited in the number of writing (around 5000 write/erase cycles per memory cell are reported for flash memory commonly use mobile devices), while no limitations exists for reading.
For this reason, mobile operating systems implements wearleveling optimization strategies consisting of writing uniformly on the whole storage disk. This limitation should be considered in designing $\mathrm{P} 2 \mathrm{P}$ applications that have to write and rewrite frequently their buffer contents.

Another significant limitation of mobile devices is bandwidth and large variability of the network connection quality. As observed in section III.B, lots of users leave a video session because of the bad viewing experience. This leads to frequent topology churn in P2P network, generating instability that worsen the downloading experience.

Last but not least, comes the problem of battery capacity. The thirst for energy in new smartphones and tablets is by far exceeding improvements in battery technology and smartphones are always short of energy. Consequently, people are increasingly cautious about battery consumption. Unfortunately, P2P applications upload information that is one of the most power-consuming operations in a mobile platform. Moreover, it is hard in a P2P platform to push the communication transceiver in idle mode, which is one of the major used strategies to save some battery resource. Therefore the use of P2P approaches for mobile video distribution is likely to reduce greatly the battery life of mobile devices that is unacceptable for nowaday users [9].

\section{CONClusion}

In this article, we analyzed the challenges a content provider has to face in order to implement and deploy a P2P mobile video distribution system over a wireless broadband network. This analysis was backed by real measurements and industry experience. Designing a P2P video distribution system for a mobile environment is quite different from designing it for a non-mobile one. In particular, the fact that mobile users usually do not see a whole video and leave it fast, the need to manage more NAT sessions and the $3 \mathrm{G}$ business model that discourage users from uploading all bring strong limitation to the development of P2P based Mobile video distribution systems over $3 \mathrm{G}$ networks. The current technology of mobile device also set some limitations on storage and battery usage.

Indeed, in mid term, some of the above issue might find solutions. For example large deployment of IPv6 can solve the issues with NAT, or new storage technologies might reduce the negative impact of rewriting information on mobile storage. However, the ensemble of the above challenges seems to be unavoidable, and as any of the above issues alone might block the deployment of a P2P based Mobile video distribution systems over $3 \mathrm{G}$ networks, we are not optimistic on the emergence of such systems in commercial mobile video distribution.

\section{REFERENCES}

[1] Cisco visual networking index, "Global mobile data traffic forecast update, 2011-2016," Technical report, Cisco, 2012.

[2] F. Dobrian, V. Sekar, A. Awan, I. Stoica, D. Joseph, A. Ganjam, J. Zhan, and H. Zhang, "Understanding the impact of video quality on user engagement," in Proc. SIGCOMM, 2011, pp. 362-373.

[3] Y. Huang, T.Z.J. Fu, D. Chiu, J.C.S. Lui, and C. Huang, "Challenges, design and analysis of a large-scale p2p-vod system," in Proc. SIGCOMM, 2008, pp. 375-388. 
[4] H. Yin, X. Liu, F. Qiu, N. Xia, C. Lin, H. Zhang, V. Sekar, and G. Min, "Inside the bird's nest: measurements of large-scale live VoD from the 2008 olympics," in Proc. Internet Measurement Conference, 2009, pp. $442-455$.

[5] H. Yu, D. Zheng, B.Y. Zhao, and W. Zheng, "Understanding user behavior in large-scale video-on-demand systems," in Proc. EuroSys, 2006, pp. 333-344.

[6] PPTV. Available: http://www.pptv.com.

[7] P. Srisuresh, B. Ford and D. Kegel, "State of Peer-to-Peer (P2P) Communication across Network Address Translators (NATs)," RFC 5128, 2008.

[8] D. Manini, R. Gaeta, and M. Sereno, "Performance Modeling of P2P File Sharing Applications," in Proc. FIRB-Perf, 2005, pp. 34-43.

[9] S. Deng, "Reducing 3G energy consumption on Mobile Devices," Master of Science thesis, MIT, Feb. 2012. 